Research paper

\title{
Exploring thermal flight responses as predictors of flight ability and geographic range size in Drosophila
}

\author{
Liana I. De Araujo, Minette Karsten, John S. Terblanche* \\ Center for Invasion Biology, Department of Conservation Ecology and Entomology, Stellenbosch University, Private Bag X1, Matieland 7602, South Africa
}

\section{A R T I C L E I N F O}

\section{Keywords:}

Thermal flight performance

Invasion

Geographic distribution

Climate change

\begin{abstract}
A B S T R A C T
Thermal flight performance curves (TFPCs) may be a useful proxy for determining dispersal on daily timescales in winged insect species. Few studies have assessed TFPCs across a range of species under standard conditions despite that they may be useful in predicting variation in performance, abundance or geographic range shifts with forecast climate variability. Indeed, the factors determining realized dispersal within and among flying insect species are generally poorly understood. To better understand how flight performance may be correlated with geographic range extent and potential latitudinal climate variability, we estimated the thermal performance curves of flight ability in 11 Drosophilidae species (in $4{ }^{\circ} \mathrm{C}$ increments across $16-28^{\circ} \mathrm{C}$ ) after standard laboratory rearing for two generations. We tested if key morphological, evolutionary or ecological factors (e.g. species identity, sex, body mass, wing loading, geographic range size) predicted traits of TFPCs (including optimum temperature, maximum performance, thermal breadth of performance) or flight ability (success/failure to fly). Although several parameters of TFPCs varied among species these were typically not statistically significant probably owing to the relatively small pool of species assessed and the limited trait variation detected. The best explanatory model of these flight responses across species included significant positive effects of test temperature and wing area. However, the rank of geographic distribution breadth and phylogeny failed to explain significant variation in most of the traits, except for thermal performance breadth, of thermal flight performance curves among these 11 species. Future studies that employ a wider range of Drosophilidae species, especially if coupled with fine-scale estimates of species' environmental niches, would be useful.
\end{abstract}

\section{Introduction}

Ambient temperature affects many traits in ectotherms including metabolism, development and locomotor performance (Gillooly et al., 2001; Ashton, 2004; Dillon and Frazier, 2006; Angilletta, 2009; Clusella-Trullas et al., 2010). Thermal performance curves (TPCs) have been argued to be useful in forecasting wholesale species range shifts (Buckley et al., 2012, 2015; Buckley and Huey, 2016; reviewed in Sinclair et al., 2016; Woods et al., 2018) in response to predicted climate change. These TPCs can also be particularly powerful in attempting to forecast more subtle or instant effects on performance (e.g. foraging or growth rates) with changing environmental conditions (e.g. Angert et al., 2011; Dell et al., 2011; Huey and Kingsolver, 2011; Barton and Terblanche, 2014; Sinclair et al., 2016; but see also discussion in Woods et al., 2018).

The influence of ambient temperature on flight responses or relative flight ability has been researched extensively across diverse insect taxa and these show typical TPCs in which there are optimal performance temperatures, and above or below these (i.e. supra- or sub-optimal), organisms experience fairly predictable declines in performance and hence fitness (Dillon and Frazier, 2006; Karlsson and Johansson, 2008; Zhang et al., 2008; Samejima and Tsubaki, 2010; Esterhuizen et al., 2014; Mason, 2017). However, prior thermal history may alter the nature of this flight response in complex ways (c.f. Drosophila melanogaster in Dillon and Frazier, 2006; and Ceratitis capitata in Esterhuizen et al., 2014). As such, studies across species need to control for, or include as a covariate, thermal history in comparative studies. Therefore, thermal flight performance curves (TFPCs) may be a useful proxy for forecasting local and regional changes in abundance and dynamics of pterygote (i.e. winged) insect species, such as those within the Diptera (e.g. Drosophilidae). To our knowledge, surprisingly few studies have assessed TFPCs across a range of species under standard laboratory conditions despite that these may be useful in predicting performance or range shifts with forecast climate patterns and which are increasingly used to this effect (e.g. Overgaard et al., 2014; Bush et al., 2016; Kellermann et al., 2018, 2019; MacLean et al., 2019; reviewed in

\footnotetext{
* Corresponding author.

E-mail addresses: minettek@sun.ac.za (M. Karsten), jst@sun.ac.za (J.S. Terblanche).
} 
Hoffmann, 2010).

We therefore aimed to better understand how insect flight performance may be influenced by ambient temperature, and in turn, correlated with geographic range extent (i.e., distribution). Thus, we estimated the TFPCs of flight ability in 11 Drosophilidae species after they had completed two generations under controlled, standardized laboratory conditions. We then tested if any major morphological or ecological factors (e.g. sex, body size, wing loading, geographic range size) could explain variation in flight responses. Moreover, we were interested in whether the distribution range (see Methods) could be predicted by the main parameters (e.g. optimum temperature, maximum performance, breadth of performance) of species-level TFPCs or any of the morphological factors measured here. Based on flight biomechanics (Dudley, 2002) we broadly expected that flies with larger wings or lower wing loading would be better fliers, though quite how such morphological variation would interact with ambient temperature is not especially well understood. We also expected that Drosophila species would generally differ in some aspects of their thermal flight response and morphology but this might be a consequence of shared evolutionary history (phylogenetic association) or varied selection pressures on diverse traits (e.g. Hoffmann et al., 2007). Specifically, we expected species that occupy broader environmental thermal niches (greater geographic range size) to have broader thermal performance curves of flight or perhaps show trait variation indicative of generalistspecialist trade-offs (Angilletta Jr, 2009).

\section{Methods}

Drosophilidae species were sampled from six locations in the Western Cape and Kwa-Zulu Natal Provinces of South Africa using bucket traps placed in shaded habitats, baited with ripe fruit (mango, grapes, lemon, oranges, figs, berries and bananas) or mushrooms in different combinations. Flies were collected by aspiration and transferred into plastic bottles filled with Bloomington's standard cornmeal diet medium (http://flystocks.bio.indiana.edu/Fly_Work/mediarecipes/bloomfood.htm) to start isofemale lines from a minimum of 4-7 field-inseminated females per species. Species were identified using the Universal cytochrome oxidase I (COI) primers (LCO1490 and HCO2198; Folmer et al., 1994) by Inqaba Biotech ${ }^{\text {TM }}$ and the South African Sugarcane Research Institute's (SASRI) biotechnology department. Five species identifications were confirmed in NCBI using BLAST (Altschul et al., 1997; de Araujo et al., 2019) and a further two species were also confirmed by a drosophilid taxonomist (Shane McEvey, Australian Museum Research Institute).

To eliminate field effects, flies captured in the respective provinces were reared at either $23^{\circ} \mathrm{C}$ (Western Cape) or $c .25^{\circ} \mathrm{C}$ (Kwa-Zulu Natal) under standard conditions for three generations. Once flies started emerging they were transferred to a new bottle with fresh diet medium at a standardized low density and replicated until F3, except for $D$. melanogaster where rearing was discontinued after two generations due to time constraints. Variation linked to aging and reproductive senescence is well known to occur in Drosophilidae (Le Bourg, 2011; Colinet et al., 2015; Hoffmann and Ross, 2018) thus to minimise these effects, all experiments were conducted using six-day old flies.

Sixty six-day old male and female drosophilids from the 3rd generation (except in the case of $D$. melanogaster) were used to test the thermal flight response in 11 Drosophilidae species. We determined performance at four different controlled temperatures $\left(16^{\circ} \mathrm{C}, 20^{\circ} \mathrm{C}\right.$, $24^{\circ} \mathrm{C}$ and $28^{\circ} \mathrm{C}$ ) using a peltier plate cooler (CP-121, TE Technology, USA) covered with a gauze swab (to prevent direct contact with the steel surface) and a temperature controller (TC-720, TE Technology, USA). In each flight temperature assay, a single $20 \mathrm{ml}$ plastic container was placed upside down on the gauze with a thermocouple (36 standard wire gauge, Type T, Omega, USA) connected to a hand-held thermometer (Fluke 52 II, Netherlands) inserted through a small hole in the side of the container. Flies were tested individually inside the $20 \mathrm{ml}$ container until $n=15$ males and $n=15$ females had been observed.

For consistency, each fly was allowed $2 \mathrm{~min}$ to equilibrate before measurement started and prodded a maximum of five times using a thin wire, which resulted in either sustained flight or no flight and were recorded as 1 and 0 respectively. 'Flight' (1) was defined as flight across the container with noticeable wing movement at least multiple times; 'no flight' (0) was recorded when the fly only jumped, walked or became immobile. Flies were prodded to measure physical ability to fly, rather than behavioural propensity to do so, as in previous work from other Diptera (Esterhuizen et al., 2014). Each fly was weighed (mg) using a Mettler UMX2 microbalance scale (CH-8606 Greifensee, Mettler-Toledo $\mathrm{GmbH}$, Laboratory \& Weighing Technologies, Switzerland) and their wing surface area $\left(\mathrm{mm}^{2}\right)$ determined. This was done by taking photographs using a Leica MZ16A auto montage microscope attached to a Leica DFC 290 fixed digital camera (Leica, Wetzlar, Germany); the wing perimeter manually outlined in ImageJ (https://imagej.nih.gov/ $\mathrm{ij} /$ ) which then calculated the area according to the scale unit of measurements provided to the software. We used the right wing for measurements in all instances except where the wing was damaged or had imperfections in which case the left wing was used. Measurements of wing area or wing loading have previously been shown to influence flight performance in a diverse range of insect species (Harrison and Roberts, 2000; Berwaerts et al., 2002; reviewed in Dudley, 2002; Harrison et al., 2012).

Sub-Saharan Drosophila species occupancy or distribution records, and thus, potential thermal microenvironment use, are poorly characterized and in many cases outdated or unknown (see e.g. De Araujo et al., 2019). Therefore, to assign a relative geographic distribution to each species, and because we could not sample species from sufficiently diverse environments within South Africa to characterize their relative distributions and thermal niches using climate databases (e.g. Overgaard et al., 2014; Bush et al., 2016), we estimated relative geographic range size using published literature and TaxoDros (www. taxodros.uzh.ch/search/dist_reg.php), South African museum records, and in consultation with Drosophila taxonomists. Rank of distribution was assigned as follows: first, we assessed if a species was found on multiple continents, and if it was known to be found in multiple diverse habitats. Second, if found on multiple continents, we scored relative rank based on similarity of habitats occupied across continents. Species distributed across different habitat types were classified as more widely-distributed and assigned a higher value and the assigned ranking value decreased as species ranges became more restricted or specialised (for example, those only found along the equatorial belt, tropical rainforests or restricted to a few locations on a single continent). A second author (JST) independently followed the same procedure and produced the same ranking of species distributions suggesting this method is reproducible and not especially subjective on the set of species we collected. The highest rank number (11) represents the most widely distributed and the lowest number (1) a narrow distribution or more habitat specialised distribution. We refer to this ranking as 'rank of geographic distribution' to serve as a proxy of thermal range likely experienced by the species across its distribution (and see discussions in Overgaard et al., 2014; Bush et al., 2016; review in Hoffmann, 2010).

We tested the effect of species, sex and test temperature on the flight responses of 11 Drosophilidae species using a GLZ with binomial distribution and logit link function (Statistica v13). The influence of individual size-related traits (body mass, wing area and wing loading) on the flight responses of species were further investigated at the different temperatures assayed using a binomial GLZ (Statistica v13). The minimum adequate model was determined by implementing the fully saturated model using the 'dredge' function in the 'MuMin' package (Bartón, 2016) in R (R Core Team, 2013) to determine what subsets or best combinations of factors most strongly influenced the estimated flight response. We ran a GLM with a binomial distribution and a logit link function, including thermal flight response as the dependent 
variable, as well as morphology (body mass, wing area and wing loading), sex, species and test temperature and their interactions as factors. Three separate sets of these models were run, once each for each of the different morphological predictors (body mass, wing area and wing loading respectively), and Akaike information criteria (AICc) used to rank models and their respective fits.

Next, TableCurve2D (http://www.sigmaplot.co.uk/products/ tablecurve2d/tablecurve2d.php) was used to fit a non-linear curve (best-fit line, scored by highest degrees-of-freedom adjusted $r^{2}$ and lowest number of model parameters) to our thermal flight performance data for each species separately. From the best-fit line (using tool 'peak: all equations' in TableCurve2D) the percentage maximum flight performance (Umax) (i.e. the proportion of population capable of flying), the optimum temperature (Topt, in ${ }^{\circ} \mathrm{C}$ ) and the performance breadth (Tbr) were extracted for each species. To determine whether Tbr, Topt, Umax, mean body mass, wing area or wing loading could explain the rank of geographic distribution of species, we ran phylogenetic generalized least-squares regression (pGLS) analyses, fitted using maximum likelihood ( $\lambda=$ ML) ('pgls' function; Freckleton et al., 2002) in 'caper' (R Core Team, 2013) and assessed if there was significant phylogenetic signal in our data. In addition, each model was run with the assumption of $\lambda=1$ and $\lambda=0$ to account for different phylogenetic relatedness. To this end, DNA sequences obtained from sequencing COI to confirm identification (described earlier) were edited using the BioEdit Sequence Alignment editor 7.0.5 (Hall, 2005) and aligned in MAFFT (Katoh et al., 2017). Sequences for two additional genes, COII and Amyrel, were downloaded from GenBank, aligned individually and then concatenated using MEGA X (Kumar et al., 2018). A Bayesian phylogeny was constructed in MRBAYES v3.2 (Ronquist et al., 2012) including 5,000,000 generations and five parallel Monte Carlo Markov chains sampled every 100th generation. We used Pagel's $\lambda$ (Pagel, 1999) as a quantitative measure for estimating phylogenetic signal. Pagel's $\lambda$ scales between zero and one, with values closer to zero showing no phylogenetic signal and values closer to 1 showing strong phylogenetic signal for the traits examined. Moreover, this allowed comparison of phylogenetic-adjusted and unadjusted (equal relatedness species, or 'star-shaped' phylogeny, where $\lambda$ is fixed $=0.000001$ ) analyses to report the best-fitting model results. We used the 'contmap' function ('phytools' package; http://www.phytools.org/) in R to visualise and graphically illustrate traits on the phylogeny generated.

\section{Results}

Thermal flight performance was not influenced by sex $(\mathrm{df}=1$, $\left.\mathrm{X}^{2}=0.009, p>.05\right)$ or species $\left(\mathrm{df}=10, \mathrm{X}^{2}=7.985, p>.05\right)$ but was influenced by experimental test temperature across all species ( $\left.\mathrm{df}=1, \mathrm{X}^{2}=10.723, p<.001\right)$. However, this general temperature effect on flight across species was largely driven by strong effects of test temperature in $D$. hydei $\left(\mathrm{X}^{2}=10.032, p<.05\right)$, and $D$. funebris $\left(X^{2}=14.050, p<.05\right)$ but not in the other species when examined in isolation. Although the thermal flight response did not differ between sexes across all species, two species showed significant sex effects at specific temperatures $\left(20^{\circ} \mathrm{C}\right.$ and $\left.28^{\circ} \mathrm{C}\right)$ whereby males and females of D. immigrans $\left(\mathrm{X}^{2}=0.6327, p>.05\right)$ and $Z$. taronus $\left(\mathrm{X}^{2}=0.00001\right.$, $p>.05$ ) responded differently (Fig. 1 ). The females of $Z$. taronus performed significantly better at $20^{\circ} \mathrm{C}$ and $28^{\circ} \mathrm{C}$ compared to their male counterparts, while $D$. immigrans females performed worse than $D$. immigrans males at these same temperatures.

To assess collinearity among variables, we assessed body mass, wing area and the interaction between them using all individuals from all species $(r=0.7699, p<.05)$. All species showed a significant positive correlation between body mass and wing area when examined individually ( $D$. hydei: $r=0.7444$; $D$. funebris: $r=0.7552 ; D$. busckii: $r=0.7335$; Z. taronus: $r=0.7037$; $Z$. vittiger: $r=0.5442$; . immigrans: $r=0.7127 ; D$. simulans: $r=0.7535 ; Z$. indianus: $r=0.4611 ; Z$. tuberculatus: $r=0.5407$; D. melanogaster: $r=0.6021$; $D$. ananassae: $r=0.6782$ ) indicating that as body mass increases so does wing area. Strong positive correlations were found between Umax and Tbr $(r=0.7364, p<.05)$, body mass and wing area $(r=0.9273$, $p<.05)$; and body mass and wing loading $(r=0.7636, p<.05)$ (Table 1). While these results indicate correlations between triats, any direction of causality, if at all, remains unclear.

Flight responses (success or failure to fly) were not influenced by the morphology of flies as measured by body mass, wing area and wing loading in all species (Table S1, Figs. S1-S3). There were no obvious or marked systematic morphological differences between flies tested and categorized into those that successfully flew ('fliers') and those that failed to fly ('failure'), when compared at each test temperature, either within or across species. Intraspecifically, D. immigrans and $Z$. taronus have large body mass variability among individuals while $D$. busckii and $Z$. indianus have small variability in body mass. While there are no obvious species trends for variability in wing area and wing loading, $D$. busckii shows the lowest variability in wing area and $Z$. indianus has the least variability in wing loading among individuals. However, when running all possible iterations of potential explanatory factors influencing thermal flight performance, including sex, species, temperature, wing area, body mass, wing loading and their interactions, the best fit model was one that included temperature and wing area but not any other predictors or interactions among them (Table 2). In other words, the Drosophila examined here generally flew more readily at warmer temperatures and if they had greater wing area.

The parameters of the TFPC extracted using TableCurve2D were further used to explore whether these potentially explain the geographic distributions of Drosophilidae based on their ranked category of distribution. We explored 32 different pGLS models (Table 3, Table S2) and, based on AICc scores and Akaike weights, single predictor models were always considered the best models of those tested. The first (best) nine models could not be significantly differentiated on the basis of model probability as AICc scores varied little among these, however the model considering the influence of Tbr on rank had the lowest AICc score. This was also the case when we followed a minimal adequate model approach. For the three best models (Tbr, Umax, Wing area) phylogenetic signal was only marginally significant for Tbr. Overall, it seems that none of the TFPC parameters could predict geographic distribution rank.

The traits of interest (Topt, Tbr, Umax, body mass, wing area and wing loading) were overlaid with phylogeny for a graphical representation of the interspecific variation (Fig. 2, Fig. S4). Drosophila busckii and $D$. funebris have the lowest Topt (Fig. 2a), which means they perform best at lower temperatures, yet they have the greatest $\mathrm{Tbr}$ (Fig. 2b). Umax variation does not appear to be clade-specific with $D$. funebris showing the highest Umax (Fig. 2c). Body mass (Fig. S4) and wing loading (Fig. S4) were the highest for Z. taronus, Z. vittiger and D. immigrans while wing area was greatest for the Zaprionus clade (Fig. 2d).

\section{Discussion}

Here we determined the thermal flight ability of 11 species in the Drosophilidae family across $16-28^{\circ} \mathrm{C}$, and measured potentially correlated morphological traits commonly associated with flight ability. We were primarily interested in 1) if there were systematic differences in thermal flight responses among species; 2) if any morphological traits (body mass, wing area, wing loading) may have explained variation in flight ability and 3) if any of the variation in TFPCs may have been explained by differences in species' geographic distributions or shared evolutionary history. Although aspects of TFPCs differed among species, such as intraspecific variation in the flight response, these differences were not always statistically significant and seldom varied systematically. Thus, among the 11 Drosophilidae species examined here, several of the findings are perhaps more novel and significant for comparative physiology. 


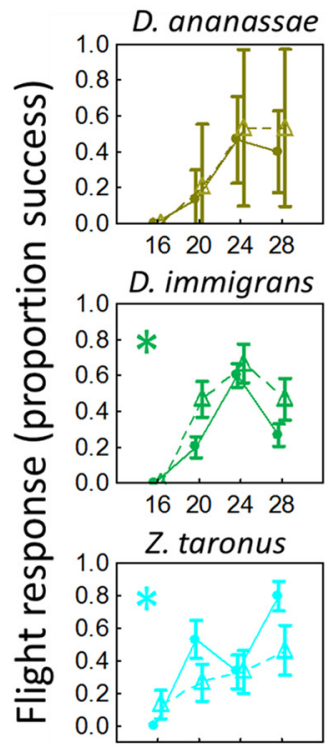

16202428
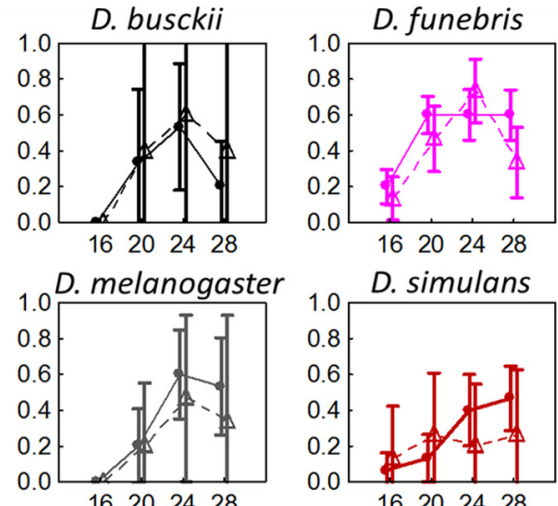

16202428
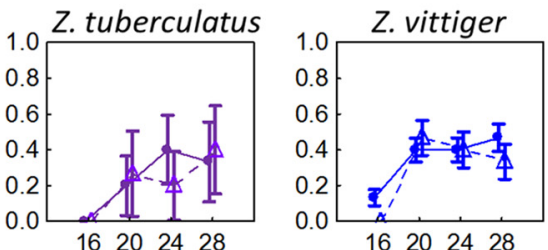

16202428

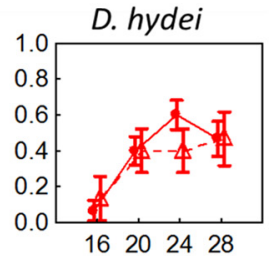

Z. indianus
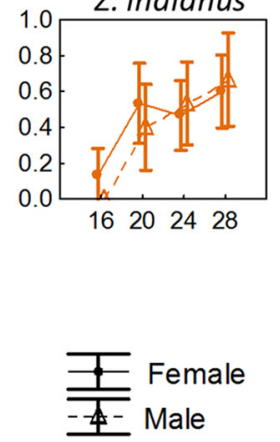

\section{Temperature $\left({ }^{\circ} \mathrm{C}\right)$}

Fig. 1. Summary results from the GLZ model (binomial data, logit link function) (showing model weighted mean $\pm 95 \%$ CI) of flight responses estimated at four test temperatures in males and females of each Drosophilidae species. The asterisk indicates a significant difference in response between the sexes.

Table 1

Results of a Spearman rank correlation test of species' geographic distribution ranking (where higher rank equals broader geographic distribution), thermal performance breadth (Tbr), optimal performance temperature (Topt, in ${ }^{\circ} \mathrm{C}$ ), the flight response scored as flight performance at Topt, (Umax), mean body mass in mg (Mb), wing area $\left(\mathrm{mm}^{2}\right)$ and wing loading $\left(\mathrm{mg} / \mathrm{mm}^{2}\right)$. Significance at $5 \%$ confidence interval $(p>.05)$ is indicated by bold font.

\begin{tabular}{|c|c|c|c|c|c|c|c|}
\hline Variable & Rank & Tbr & Topt $\left({ }^{\circ} \mathrm{C}\right)$ & Umax & $\mathrm{Mb}(\mathrm{mg})$ & Wing area $\left(\mathrm{mm}^{2}\right)$ & Wing loading $\left(\mathrm{mg} / \mathrm{mm}^{2}\right)$ \\
\hline Rank & 1.000 & 0.073 & -0.092 & 0.146 & -0.255 & -0.264 & 0.027 \\
\hline Tbr & & 1.000 & -0.422 & 0.736 & 0.418 & 0.582 & 0.182 \\
\hline Topt $\left({ }^{\circ} \mathrm{C}\right)$ & & & 1.000 & -0.193 & -0.128 & -0.275 & -0.294 \\
\hline Umax & & & & 1.000 & 0.136 & 0.300 & 0.073 \\
\hline $\mathrm{Mb}(\mathrm{mg})$ & & & & & 1.000 & 0.927 & 0.764 \\
\hline Wing area $\left(\mathrm{mm}^{2}\right)$ & & & & & & 1.000 & 0.564 \\
\hline Wing loading $\left(\mathrm{mg} / \mathrm{mm}^{2}\right)$ & & & & & & & 1.000 \\
\hline
\end{tabular}

Table 2

Summary of the coefficients and results for the overall best-fit model for Drosophilidae flight response obtained from the 'dredge' function in the 'MuMin' package in R. All effects presented below are significant.

\begin{tabular}{lllll}
\hline & Estimate & Std. error & $\mathrm{z}$ value & $\mathrm{p}$ \\
\hline Intercept & -4.0644 & 0.3355 & -12.113 & $<\mathbf{0 . 0 0 1}$ \\
Temperature & 0.1070 & 0.0116 & 9.226 & $<\mathbf{0 . 0 0 1}$ \\
Wing area & 0.2151 & 0.0731 & 2.941 & $<\mathbf{0 . 0 1}$ \\
\hline
\end{tabular}

First, the novel thermal flight performance curves generated for each species provide unique data that, according to our knowledge, are not readily available in the literature. Thermal locomotor responses have been determined in a few selected Drosophila species across diverse methodological approaches (Gilchrist et al., 1997; Gibert et al., 2001; Dillon and Frazier, 2006; Latimer et al., 2011) but the thermal flight response of most of the Drosophila species used in our study has not previously been determined. The trend found among our species was that flight was less common at cooler temperatures (and vice versa) and perhaps is largely expected given the foregoing literature (e.g. Dillon and Frazier, 2006 for D. melanogaster). Of the temperatures tested here, most species reached their thermal optimal performance at c. $24^{\circ} \mathrm{C}$, which resulted in fewer responses at $28^{\circ} \mathrm{C}$; this trend is most visible in both sexes of $D$. busckii, $D$. immigrans, $D$. melanogaster, females of $D$. hydei, $D$. ananassae and Z. tuberculatus, and $D$. funebris males. The optimal temperature for some species (Z. taronus, D. simulans females, $D$. hydei males, $Z$. tuberculatus males and $Z$. indianus males) appeared to have not yet been reached at $28^{\circ} \mathrm{C}$ and the TFPC therefore did not taper off or show obvious declines in the proportion of flying individuals.

The different parameters (Topt, Tbr, Umax) of the thermal flight performance curve were extracted and considered at the species level, and given our study's experimental design and methodological approach, could not be determined at the individual level within each species. In contrast with results of Sørensen et al. (2018), we found no significant positive correlation between Umax and Topt, but we did find this in the case of Tbr and Umax. The Umax, Tbr and Topt was not significantly different between species and those with the lowest (specialised) ranking had the highest Topt, which corresponds with their known climatic Afrotropical distributions (Z. taronus, Z. vittiger and Z. tuberculatus) (Bächli, 2018). However, this is likely to be driven by the lower statistical power in our study compared to that of Sørensen et al. (2018) who used a much greater compilation of species. These findings may provide evidence of interspecific generalist-specialist trade-offs (Angilletta Jr, 2009) of thermal flight performance curves that warrants further investigation. Second, morphological traits are frequently correlated with locomotor ability (De Bie et al., 2012; Reim et al., 2018), and the same is true for flight ability in insects (e.g. Berwaerts et al., 2002; Dudley, 2002; Van Dyck and Wiklund, 2002; Esterhuizen et al., 2014; Steyn et al., 2016). We therefore considered body mass, wing area and wing loading in detail here. These traits were however not 
Table 3

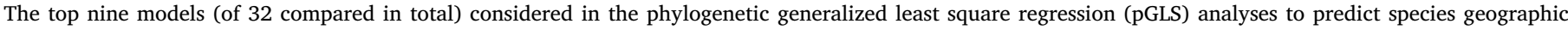

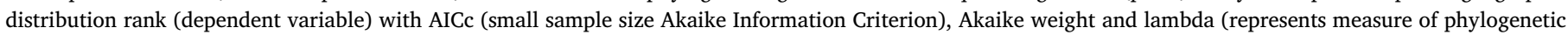
strength) values.

\begin{tabular}{|c|c|c|c|c|c|c|c|c|c|c|c|c|c|}
\hline Statistical model & $\begin{array}{l}\text { Evolutionary } \\
\text { model }\end{array}$ & Intercept & Tbr & Umax & Wing area & Topt & $\mathrm{Mb}$ & $\begin{array}{l}\text { Wing } \\
\text { loading }\end{array}$ & $\log (\mathrm{Mb})$ & $\begin{array}{l}\log \text { (Wing } \\
\text { area) }\end{array}$ & $\begin{array}{l}\text { log (Wing } \\
\text { loading) }\end{array}$ & AICc & $\begin{array}{l}\text { Akaike } \\
\text { weights }\end{array}$ \\
\hline Tbr & $\lambda=\mathrm{ML}$ & 0.943 & 1.103 & & & & & & & & & 56.651 & 0.100 \\
\hline Tbr & $\lambda=1$ & 0.943 & 1.103 & & & & & & & & & 56.651 & 0.100 \\
\hline Tbr & $\lambda=0.000001$ & 3.999 & 0.392 & & & & & & & & & 61.633 & 0.008 \\
\hline Umax & $\lambda=\mathrm{ML}$ & 4.227 & & 3.672 & & & & & & & & 58.095 & 0.049 \\
\hline Umax & $\lambda=1$ & 4.227 & & 3.672 & & & & & & & & 58.095 & 0.049 \\
\hline Umax & $\lambda=0.000001$ & 1.521 & & 7.807 & & & & & & & & 61.081 & 0.011 \\
\hline Wing_area & $\lambda=\mathrm{ML}$ & 4.477 & & & 0.747 & & & & & & & 58.248 & 0.045 \\
\hline Wing_area & $\lambda=1$ & 4.477 & & & 0.747 & & & & & & & 58.248 & 0.045 \\
\hline Wing_area & $\lambda=0.000001$ & 8.126 & & & -0.988 & & & & & & & 61.483 & 0.009 \\
\hline Topt & $\lambda=\mathrm{ML}$ & 9.683 & & & & -0.134 & & & & & & 58.251 & 0.045 \\
\hline Topt & $\lambda=1$ & 9.683 & & & & -0.134 & & & & & & 58.251 & 0.045 \\
\hline Topt & $\lambda=0.000001$ & 17.982 & & & & -0.479 & & & & & & 60.770 & 0.013 \\
\hline $\mathrm{Mb}$ & $\lambda=\mathrm{ML}$ & 6.292 & & & & & 0.029 & & & & & 58.480 & 0.040 \\
\hline $\mathrm{Mb}$ & $\lambda=1$ & 6.292 & & & & & 0.029 & & & & & 58.480 & 0.040 \\
\hline $\mathrm{Mb}$ & $\lambda=0.000001$ & 5.789 & & & & & 0.002 & & & & & 61.800 & 0.008 \\
\hline Wing_loading & $\lambda=\mathrm{ML}$ & 6.975 & & & & & & -0.754 & & & & 58.406 & 0.042 \\
\hline Wing_loading & $\lambda=1$ & 6.975 & & & & & & -0.754 & & & & 58.406 & 0.042 \\
\hline Wing_loading & $\lambda=0.000001$ & 4.893 & & & & & & 1.155 & & & & 61.710 & 0.008 \\
\hline $\log (\mathrm{Mb})$ & $\lambda=\mathrm{ML}$ & 6.493 & & & & & & & -0.209 & & & 58.465 & 0.040 \\
\hline $\log (\mathrm{Mb})$ & $\lambda=1$ & 6.493 & & & & & & & -0.209 & & & 58.465 & 0.040 \\
\hline $\log (\mathrm{Mb})$ & $\lambda=0.000001$ & 5.970 & & & & & & & -0.337 & & & 61.766 & 0.008 \\
\hline log(Wing_area) & $\lambda=\mathrm{ML}$ & 5.393 & & & & & & & & 1.068 & & 58.368 & 0.042 \\
\hline log(Wing_area) & $\lambda=1$ & 5.393 & & & & & & & & 1.068 & & 58.368 & 0.042 \\
\hline log(Wing_area) & $\lambda=0.000001$ & 7.798 & & & & & & & & -2.420 & & 61.318 & 0.010 \\
\hline log(Wing_loading) & $\lambda=\mathrm{ML}$ & 6.190 & & & & & & & & & -0.697 & 58.354 & 0.043 \\
\hline log(Wing_loading) & $\lambda=1$ & 6.190 & & & & & & & & & -0.697 & 58.354 & 0.043 \\
\hline log(Wing_loading) & $\lambda=0.000001$ & 6.031 & & & & & & & & & 0.798 & 61.723 & 0.008 \\
\hline
\end{tabular}

found to be strongly associated with thermal flight responses, a finding that is in keeping with some earlier studies. For example, Dillon and Frazier (2006) found that body mass was not significantly associated with flight ability in D. melanogaster while Reim et al. (2018) found wing loading, among other traits, to have a significant influence on flight ability in a butterfly species (Lycaena tityrus). Mass and wing area are positively correlated, a result which was largely expected, however, that the best model among several tested was one that used temperature
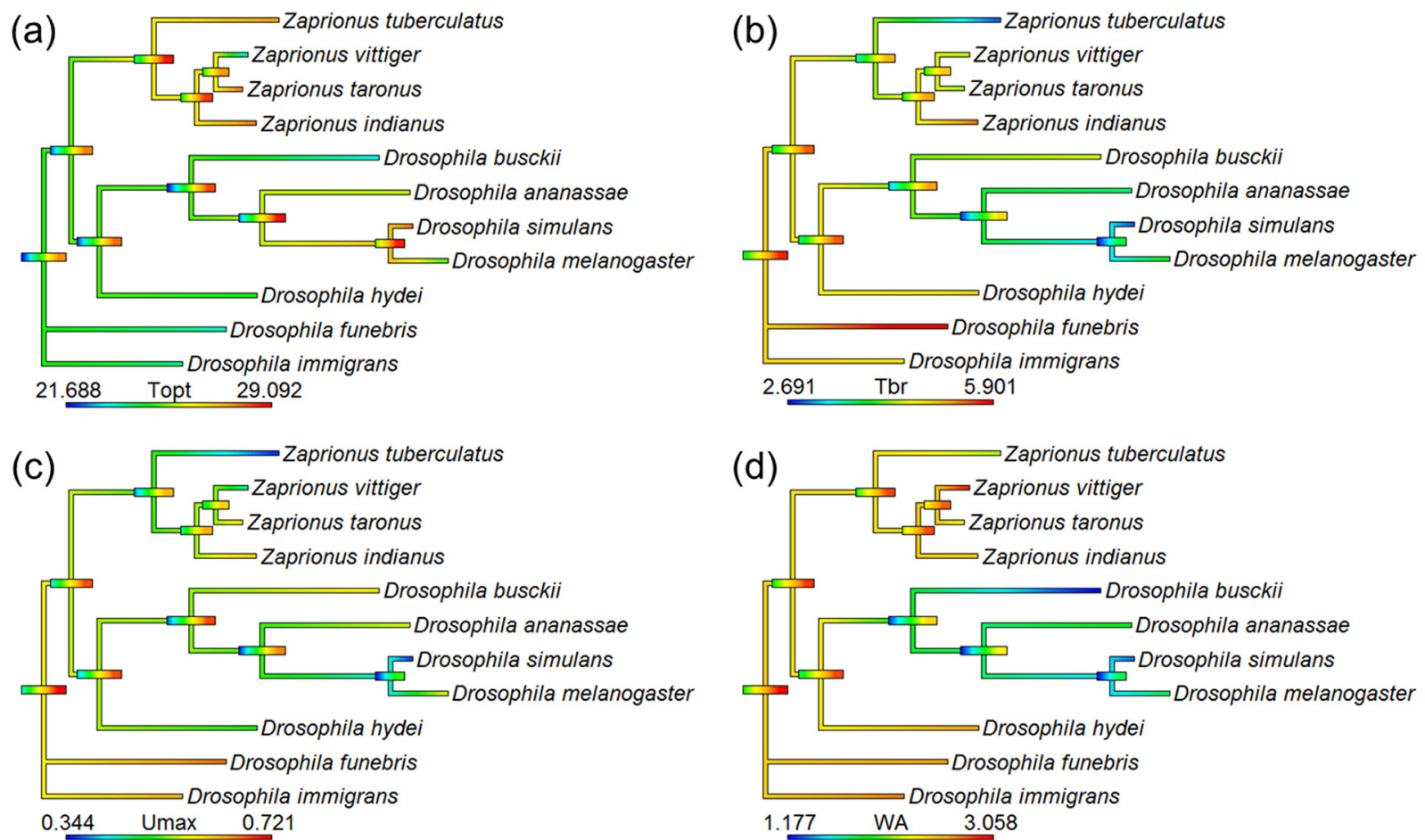

Fig. 2. Phylogenetic tree of 11 Drosophilidae species included in our study overlaid with their a) optimal thermal temperature of flight performance (Topt), b) thermal performance breadth of flight (Tbr), c) Umax of flight and d) wing area (WA). The bars at the nodes represent the standard error at the nodes while colours towards the red end of the scale indicate higher trait values compared to the blue end of the scale. Illustrations were made in R ('contmap' function in 'phytools' package). 
and wing area as predictors, is perhaps a more unusual finding as wing area is not typically considered to be a primary determinant of flight ability.

Finally, the rank of geographic range extent (or breadth of geographic distribution) as a proxy of thermal variability for the species, was poorly predicted by a range of TFPC parameters we extracted. This result is perhaps unexpected given recent studies using thermal performance curves based on other diverse traits (see e.g. Overgaard et al., 2014; Bush et al., 2016). Moreover, phylogenetic history failed to contribute meaningful variation to explain such flight responses across the thermal range employed here in all traits except in Tbr. However, this could be explained by two main limitations of our study: 1) that the species we have compiled are perhaps all closely-related relative to the diversity of the Drosophilidae and 2) we failed to rear truly specialised species in this study and together these may have biased our results towards finding a weak phylogenetic signal. Some studies suggest Type I errors can be common while Type II errors are less likely in phylogenetic independent contrasts (Mazel et al., 2016). Recent studies of other traits (e.g. MacLean et al., 2019; Kellermann et al., 2019) have also suggested thermal optima of performance curves are quite conserved and similar across species of Drosophila so this result is perhaps not surprising. However, given that diverse traits often yield contrasting results in interspecific comparisons further work is needed to probe the source of this lack of variation. Limited existing information on these traits across the Drosophilidae phylogeny precludes more detailed comparison with other studies. Indeed, there is remarkably little known about the impacts of various physiological factors that contribute to current geographic distributions or future range expansions or invasions (Hoffmann, 2010) although studies continue to address these issues in increasing detail (e.g. Overgaard et al., 2014; Bush et al., 2016).

This study provides important baseline information on species thermal flight performance and attempts to close the gap between flight performance and dispersal ability under field conditions in the Drosophilidae. According to the TFPCs we estimated here, all species fly well in warmer, albeit not injurious, conditions. Given concerns surrounding the increasing frequency of heat waves and mean temperatures with climate change, this work could be expanded to explore these factors and their impacts on flight ability or explore the geographic consequences of subtle shifts in the shapes of TFPCs (e.g. Woods et al., 2018). Dillon et al. (2009) emphasised that developmental temperature can influence the thermal preference of some species (D. melanogaster) yet have no effect on other species (e.g. D. simulans). The preferred temperature of Drosophilidae can also vary across stages of the lifecycle or with adult age (Yamamoto and Ohba, 1982, 1984; Sayeed and Benzer, 1996). Thus, future work could expand the traits across a broader age range and consider responses to thermal rearing conditions to document phenotypic plasticity of these TFPCs.

To conclude, we found little systematic variation in thermal flight performance curves across the 11 species tested within the Drosophilidae family and none of the flight parameters we considered could predict rank of geographic distribution with great confidence, except perhaps to a limited extent for Tbr. In our study, the best predictors of flight ability among species were ambient temperature and wing area. Future work investigating the thermal flight performance across a greater diversity of Drosophilidae and under a wider range of conditions, coupled with estimates of microclimate conditions experienced by diverse species, would be valuable.

\section{Acknowledgements}

We are grateful to Tania Vergnani and Jan Coetzee, Matroosberg Private Nature Reserve, South African Sugarcane Research Institute (SASRI) and Stellenbosch University Botanical Gardens that allowed sampling on their property. SASRI provided assistance with sequencing and species identification. Dr. Desmond Conlong assisted with trapping in KZN and Dr. Shane McEvey kindly assisted with species identifications. We are grateful for constructive, insightful comments by the anonymous referees that helped improve the work.

\section{Funding}

This research was supported by the DST-NRF Centre of Excellence for Invasion Biology (CIIB), Stellenbosch University, South Africa. MK was supported by HORTGRO and SATI.

\section{Declaration of Competing Interests}

There are no conflicts of interest that we are aware of. All authors agree to this submission and have contributed substantially to the work presented.

\section{Appendix A. Supplementary data}

Supplementary data to this article can be found online at https:// doi.org/10.1016/j.cbpa.2019.110532.

\section{References}

Angert, A.L., Crozier, L.G., Rissler, L.J., Gilman, S.E., Tewksbury, J.J., Chunco, A.J., 2011. Do species' traits predict recent shifts at expanding range edges? Ecol. Lett. 14, $677-689$.

Angilletta Jr., M.J., 2009. Thermal Adaptation: A Theoretical and Empirical Synthesis. Oxford University Press, New York.

Ashton, K.G., 2004. Sensitivity of intraspecific latitudinal clines of body size for tetrapods to sampling, latitude and body size. Integr. Comp. Biol. 44, 403-412.

Altschul, S.F., Madden, T.L., Schäffer, A.A., Zhang, J., Zhang, Z., Miller, W., Lipman, D.J., 1997. Gapped BLAST and PSI-BLAST: a new generation of protein database search programs. Nucleic Acids Res. 25, 3389-3402

Bächli, G., 2018. Drosophila Biodiversity. The Database on Taxonomy of Drosophilidae (TaxoDros), Zürich, Switzerland. https://www.taxodros.uzh.ch/index.php.

Bartón, K., 2016. R-Package 'MuMIn', Model Selection and Model Averaging Based on Information Criteria (AICc and Alike).

Barton, M.G., Terblanche, J.S., 2014. Predicting performance and survival across topographically heterogeneous landscapes: the global pest insect Helicoverpa armigera (Hübner, 1808) (Lepidoptera: Noctuidae). Austral Entomol. 53, 249-258.

Berwaerts, K., Van Dyck, H., Aerts, P., 2002. Does flight morphology relate to flight performance? An experimental test with the butterfly Pararge aegeria. Funct. Ecol. 16, 484-491.

Buckley, L.B., Ehrenberger, J.C., Angilletta, M.J., 2015. Thermoregulatory behaviour limits local adaptation of thermal niches and confers sensitivity to climate change. Funct. Ecol. 29, 1038-1047.

Buckley, L.B., Huey, R.B., 2016. How extreme temperatures impact organisms and the evolution of their thermal tolerance. Integr. Comp. Biol. 56, 98-109.

Buckley, L.B., Hurlbert, A.H., Jetz, W., 2012. Broad-scale ecological implications of ectothermy and endothermy in changing environments. Glob. Ecol. Biogeogr. 21, 873-885.

Bush, A., Mokany, K., Catullo, R., Hoffmann, A., Kellermann, V., Sgrò, C., McEvey, S., Ferrier, S., 2016. Incorporating evolutionary adaptation in species distribution modelling reduces projected vulnerability to climate change. Ecol. Lett. 19, 1468-1478.

Clusella-Trullas, S., Terblanche, J.S., Chown, S.L., 2010. Phenotypic plasticity of locomotion performance in the seed harvester Messor capensis (Formicidae). Physiol. Biochem. Zool. 83, 519-530.

Colinet, H., Chertemps, T., Boulogne, I., Siaussat, D., 2015. Age-related decline of abiotic stress tolerance in young Drosophila melanogaster adults. J. Gerontol. A Biol. Sci. Med. Sci. 71, 1574-1580.

De Araujo, L.I., Karsten, M., Terblanche, J.S., 2019. Three new Drosophilidae species records for South Africa. Bothalia 49, a2429.

De Bie, T., De Meester, L., Brendonck, L., Martens, K., Goddeeris, B., Ercken, D., Hampel, H., Denys, L., Vanhecke, L., Van Der Gucht, K., Van Wichelen, J., 2012. Body size and dispersal mode as key traits determining metacommunity structure of aquatic organisms. Ecol. Lett. 15, 740-747.

Dell, A.I., Pawar, S., Savage, V.M., 2011. Systematic variation in the temperature dependence of physiological and ecological traits. Proc. Natl. Acad. Sci. 108, 10591-10596.

Dillon, M.E., Frazier, M.R., 2006. Drosophila melanogaster locomotion in cold thin air. J. Exp. Biol. 209, 364-371.

Dillon, M.E., Wang, G., Garrity, P.A., Huey, R.B., 2009. Thermal preference in Drosophila. J. Therm. Biol. 34, 109-119.

Dudley, R., 2002. The Biomechanics of Insect Flight: Form, Function, Evolution. Princeton University Press, New Jersey.

Esterhuizen, N., Clusella-Trullas, S., Van Daalen, C.E., Schoombie, R.E., Boardman, L., Terblanche, J.S., 2014. Effects of within-generation thermal history on flight performance of Ceratitis capitata: colder is better. J. Exp. Biol. 217, 3545-3556. 
Folmer, O., Black, M., Hoeh, W., Lutz, R., Vrijenhoek, R., 1994. DNA primers for amplification of mitochondrial cytochrome c oxidase subunit I from diverse metazoan invertebrates. Mol. Mar. Biol. Biotech. 3, 294-299.

Freckleton, R.P., Harvey, P.H., Pagel, M., 2002. Phylogenetic analysis and comparative data: a test and review of evidence. Am. Nat. 160, 712-726.

Gibert, P., Huey, R.B., Gilchrist, G.W., 2001. Locomotor performance of Drosophila melanogaster: interactions among developmental and adult temperatures, age, and geography. Evol. 55, 205-209.

Gilchrist, G.W., Huey, R.B., Partridge, L., 1997. Thermal sensitivity of Drosophila melanogaster: evolutionary responses of adults and eggs to laboratory natural selection at different temperatures. Physiol. Zool. 70, 403-414.

Gillooly, J.F., Brown, J.H., West, G.B., Savage, V.M., Charnov, E.L., 2001. Effects of size and temperature on metabolic rate. Sci. 293, 2248-2251.

Hall, T., 2005. BioEdit, Biological Sequence Alignment Editor for Win95/98/NT/2K/XP. California, USA. http://www.mbio.ncsu.edu/BioEdit/bioedit.html.

Harrison, J.F., Roberts, S.P., 2000. Flight respiration and energetics. Annu. Rev. Physiol. 62, 179-205.

Harrison, J.F., Woods, H.A., Roberts, S.P., 2012. Ecological and Environmental Physiology of Insects. Oxford University Press, New York.

Hoffmann, A.A., 2010. Physiological climatic limits in Drosophila: patterns and implications. J. Exp. Biol. 213, 870-880.

Hoffmann, A.A., Ratna, E., Sgro, C.M., Barton, M., Blacket, M., Hallas, R., De Garis, S., Weeks, A.R., 2007. Antagonistic selection between adult thorax and wing size in field released Drosophila melanogaster independent of thermal conditions. J. Evol. Biol. 20, 2219-2227.

Hoffmann, A.A., Ross, P.A., 2018. Rates and patterns of laboratory adaptation in (mostly) insects. J. Econ. Entomol. 111, 501-509.

Huey, R.B., Kingsolver, J.G., 2011. Variation in universal temperature dependence of biological rates. Proc. Natl. Acad. Sci. 108, 10377-10378.

Karlsson, B., Johansson, A., 2008. Seasonal polyphenism and developmental trade-offs between flight ability and egg laying in a pierid butterfly. Proc. Roy. Soc. Lond. B. Biol. 275, 2131-2136.

Katoh, K., Rozewicki, J., Yamada, K.D., 2017. MAFFT online service: multiple sequence alignment, interactive sequence choice and visualization. Brief. Bioinform. 1-7.

Kellermann, V., Chown, S.L., Schou, M.F., Aitkenhead, I., Janion-Scheepers, C., Clemson, A., Scott, M.T., Sgrò, C.M., 2019. Comparing thermal performance curves across traits: how consistent are they? J. Exp. Biol. 222, jeb193433.

Kellermann, V., Hoffmann, A.A., Overgaard, J., Loeschcke, V., Sgrò, C.M., 2018. Plasticity for desiccation tolerance across Drosophila species is affected by phylogeny and climate in complex ways. Proc. Roy. Soc. Lond. B. Biol. 285, 20180048.

Kumar, S., Stecher, G., Li, M., Knyaz, C., Tamura, K., 2018. MEGA X: molecular evolutionary genetics analysis across computing platforms. Mol. Biol. Evol. 35, 1547-1549.

Latimer, C.A.L., Wilson, R.S., Chenoweth, S.F., 2011. Quantitative genetic variation for thermal performance curves within and among natural populations of Drosophila serrata. J. Evol. Biol. 24, 965-975.

Le Bourg, É., 2011. Using Drosophila melanogaster to study the positive effects of mild stress on aging. Exp. Gerontol. 46, 345-348.

MacLean, H., Sørensen, J.G., Kristensen, T.N., Loeschcke, V., Beedholm, K., Kellermann,
V., Overgaard, J., 2019. Evolution and plasticity of thermal performance: an analysis of variation in thermal tolerance and fitness in 22 Drosophila species. Philos. T. Roy. Soc. B. 374,20180548

Mason, N.A., 2017. Effects of wind, ambient temperature and sun position on damselfly flight activity and perch orientation. Anim. Behav. 124, 175-181.

Mazel, F., Davies, T.J., Georges, D., Lavergne, S., Thuiller, W., Peres-Neto, P.R., 2016. Improving phylogenetic regression under complex evolutionary models. Ecology 97, 286-293.

Overgaard, J., Kearney, M.R., Hoffmann, A.A., 2014. Sensitivity to thermal extremes in Australian Drosophila implies similar impacts of climate change on the distribution of widespread and tropical species. Glob. Chang. Biol. 20, 1738-1750.

Pagel, M., 1999. Inferring the historical patterns of biological evolution. Nature 401, 877.

R Core Team, 2013. R: A Language and Environment for Statistical Computing. R Foundation for Statistical Computing, Vienna, Austria. http://www.R-project.org.

Reim, E., Baguette, M., Günter, F., Fischer, K., 2018. Emigration propensity and flight performance are decoupled in a butterfly. Ecosphere 9, e02502.

Ronquist, F., Teslenko, M., Van Der Mark, P., Ayres, D.L., Darling, A., Höhna, S., Larget, B., Liu, L., Suchard, M.A., Huelsenbeck, J.P., 2012. MrBayes 3.2: efficient Bayesian phylogenetic inference and model choice across a large model space. Syst. Biol. 61, 539-542.

Samejima, Y., Tsubaki, Y., 2010. Body temperature and body size affect flight performance in a damselfly. Behav. Ecol. Sociobiol. 64, 685-692.

Sayeed, O., Benzer, S., 1996. Behavioral genetics of thermosensation and hygrosensation in Drosophila. Proc. Natl. Acad. Sci. 93, 6079-6084.

Sinclair, B.J., Marshall, K.E., Sewell, M.A., Levesque, D.L., Willett, C.S., Slotsbo, S., Dong, Y., Harley, C.D., Marshall, D.J., Helmuth, B.S., Huey, R.B., 2016. Can we predict ectotherm responses to climate change using thermal performance curves and body temperatures? Ecol. Lett. 19, 1372-1385.

Sørensen, J.G., White, C.R., Duffy, G.A., Chown, S.L., 2018. A widespread thermodynamic effect, but maintenance of biological rates through space across life's major domains. Proc. Roy. Soc. Lond. B. Biol. 285, 20181775.

Steyn, V.M., Mitchell, K.A., Terblanche, J.S., 2016. Dispersal propensity, but not flight performance, explains variation in dispersal ability. Proc. Roy. Soc. Lond. B. Biol. 283, 20160905.

Van Dyck, H., Wiklund, C., 2002. Seasonal butterfly design: morphological plasticity among three developmental pathways relative to sex, flight and thermoregulation. J. Evol. Biol. 15, 216-225.

Woods, H.A., Kingsolver, J.G., Fey, S.B., Vasseur, D.A., 2018. Uncertainty in geographical estimates of performance and fitness. Methods Ecol. Evol. 9, 1996-2008.

Yamamoto, A., Ohba, S., 1982. Strategic differences in thermal adaptation between 2 Drosophila species, D. virilis and D. immigrans. Oecologia 52, 333-339.

Yamamoto, A., Ohba, S., 1984. Temperature preferences of 11 Drosophila species from Japan-the relationship between preferred temperature and some ecological characteristics in their natural habitats. Zool. Sci. 1, 631-640.

Zhang, Y., Wang, L., Wu, K., Wyckhuys, K.A., Heimpel, G.E., 2008. Flight performance of the soybean aphid, Aphis glycines (Hemiptera: Aphididae) under different temperature and humidity regimens. Environ. Entomol. 37, 301-306. 